\title{
Enumerating Optimal Quantum Circuits using Spectral Classification
}

\author{
Giulia Meuli* Mathias Soeken*† Martin Roetteler ${ }^{\dagger}$ Giovanni De Micheli* \\ ${ }^{*}$ LSI, EPFL, Lausanne, Switzerland $\quad{ }^{\dagger}$ Microsoft, Redmond, WA, USA
}

\begin{abstract}
This work targets fault-tolerant quantum computing and focuses on the problem of mapping reversible circuits into the Clifford $+T$ quantum gate library. We present an automatically-generated database containing minimal-cost quantum circuits for Boolean functions up to 5 inputs. The database contains three circuits for each spectral-equivalent class representative, which are respectively optimized for the $T$-count, the $T$-depth, and the number of qubits. We show that any Boolean function can be derived from the implementation of its class representative without increasing any of the stated cost functions.
\end{abstract}

\section{INTRODUCTION}

Few quantum systems have recently been developed by technology companies and their academic partners [1]-[3]. Current fabrication technologies only enable systems with few noisy qubits, referred to as Noisy-Intermediate-Scale Quantum (NISQ) systems. While the capabilities of such systems may suffice in some specific applications, their size and noise levels do not allow to compute fault-tolerantly, i.e., using quantum error-correcting codes. As the technology is developing, it is believed that fault-tolerant quantum computing will revolutionize the way computation is performed through the development of quantum algorithms, e.g., [4]-[6], which can break the lower bound complexities of their classical counterparts.

Quantum algorithms often require the computation of large classical logic functions to be performed directly on the quantum system. Such logic functions are usually specified using a high-level description language that has to be mapped into native and application-specific operations. This process is called quantum compilation. In fault-tolerant quantum computing, native operations are grouped into the universal Clifford $+T$ library. This library contains the $T$ gate, which is very expensive to be implemented fault-tolerantly and is often the only one accounted for when estimating the cost of a quantum algorithm [7], [8]. In this setting, compilation aims at minimizing both the $T$-count, which is the number of $T$ gates and the $T$-depth, which is the maximum number of $T$ gates that cannot be performed in parallel [9]. Finally, compilation targets the minimization of the total number of qubits of the resulting quantum circuit.

In this work, we present a database of quantum circuits for all the representatives of the spectral-equivalent classes of Boolean functions with 4 and 5 inputs. To generate the circuits, we use three different compilation algorithms: two existing ones, designed to minimize the $T$-count and the number of qubits, and a new algorithm to minimize the $T$-depth.

The database can be used to implement any Boolean function. First, any 4- and 5-input function can be directly generated from the spectral-equivalent entry without using any additional qubit or $T$ gate. Second, larger Boolean functions can be automatically compiled from the database exploiting hierarchical methods as, e.g., the one proposed in [10].

\section{Preliminaries}

\section{A. Boolean functions}

A Boolean function over $n$ variables is defined as $f: \mathbb{B}^{n} \rightarrow$ $\mathbb{B}$, where $\mathbb{B}=\{0,1\}$. A Boolean function can be represented by its truth table, which is a bitstring $b_{2^{n}-1} b_{2^{n}-2} \ldots b_{0}$ of size $2^{n}$ where $b_{x}=f\left(x_{1}, \ldots, x_{n}\right)$ when $x=\left(x_{1} x_{2} \ldots x_{n}\right)_{2}$. For large functions, it is convenient to use a hexadecimal encoding of the bitstring.

Example 1: The truth table of the majority-of-three function $\left\langle x_{1} x_{2} x_{3}\right\rangle$ is 11101000 or ${ }^{\#} \mathbf{e} 8$ in hexadecimal encoding.

Every Boolean function can also be represented in terms of an exclusive sum-of-products (ESOP) expression. This representation is not unique and many heuristic and exact minimization methods have been proposed [11]-[14].

\section{B. Logic networks}

Multi-level logic networks are scalable representations of Boolean functions. A logic network is represented by a graph in which each node performs a Boolean operation and edges define data dependencies. The inputs of the function are the primary inputs of the graph. Networks are characterized by their size, i.e., the number of nodes, and by their depth, i.e., the number of levels in the graph. Two nodes are in the same level if they have the same maximum distance from the primary inputs. According to the characteristics of the network, we define different graph representations. In this work, we use Xor-And-inverter Graphs (XAG), in which nodes implement the 2 -input XOR, the 2-input AND. and inversion. Fig. 2 shows the XAG network for the majority-of-three function ${ }^{\#} e$, where dashed edges represent inversion.

\section{Reversible circuits}

The automatic compilation of a Boolean function $f$ usually proceeds by embedding $f$ in a reversible function.

Definition 1 (Reversible Boolean function): A multi-output Boolean function $f: B^{n} \rightarrow B^{m}$ is reversible, iff $f$ is a bijection: each input pattern uniquely maps to an output pattern. A reversible function is implemented using reversible gates; in particular, we define the single-target gate and the multiple-control Toffoli gate.

Definition 2 (Single-target gate): Let $c: \mathbb{B}^{k} \rightarrow \mathbb{B}$ be a Boolean function, called the control function. Also, let $C=$ $\left\{c_{1}, \ldots, c_{k}\right\}$ be a set of control lines and let $t \notin C$ be a target 

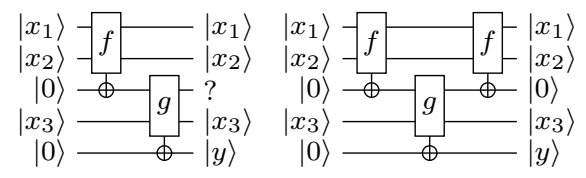

Fig. 1. (a) The function $y=g\left(f\left(x_{1}, x_{2}\right)\right)$ is computed using two single-target gates. An unknown intermediate result is generated. (b) Garbage-free circuit where the intermediate result has been uncomputed by applying $f$ twice.

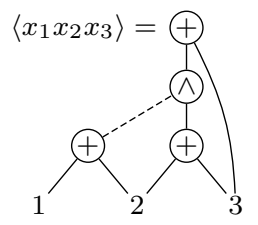

Fig. 2. An XAG for the majority of 3-inputs.

line. Then the single-target gate $\mathrm{T}_{c}(C, t): \mathrm{B}^{n} \rightarrow \mathrm{B}^{n}$ is a reversible Boolean function which maps

$$
\begin{array}{ll}
\left(x_{1}, \ldots, x_{n}\right) \mapsto\left(x_{1}^{\prime} \ldots, x_{n}^{\prime}\right) & \text { where } \\
x_{i}^{\prime}= \begin{cases}x_{i} & \text { if } i \neq t, \\
x_{t} \oplus c\left(x_{c_{1}}, \ldots, x_{c_{k}}\right) & \text { otherwise. }\end{cases}
\end{array}
$$

In other words, it inverts the target if and only if the control function evaluates to true.

Definition 3 (Multiple-control Toffoli gate): A multiple-control Toffoli gate is a single-target gate $\mathrm{T}_{c}(C, t)$ whose function is a single product term.

Quantum compilation requires reversible circuits to be garbage-free, which means that all intermediate results need to be uncomputed. This is due to the fact that quantum circuits are run on a superposition of different inputs (quantum parallelism), and measuring and resetting garbage bits can collapse the quantum state that encodes the data. Fig. 1 shows a reversible circuit in which the intermediate state is uncomputed by repeating a single-target gate.

\section{Quantum circuits}

Quantum circuits describe a sequence of operations, represented by quantum gates. In fault-tolerant quantum computing, we consider the Clifford $+T$ universal library which consists of the CNOT gate, the Hadamard gate, abbreviated $H$, as well as the $T$ gate, and its inverse $T^{\dagger}$. The $T$ gate is sufficiently expensive that it is customary to neglect all other gates when costing a quantum algorithm. The CNOT gate is a 2-qubit gate which inverts its target if the control qubit is in the one state. Classically, it would correspond to a single-control Toffoli gate. A CNOT gate with zero controls is an X gate. For more details on quantum gates, we refer the reader to [15].

Automatic quantum compilation requires quantum implementations of the reversible gates. The 2-control Toffoli gate has a Clifford $+T$ implementation that requires $7 T$ gates [7], which is optimum [8], [16]:

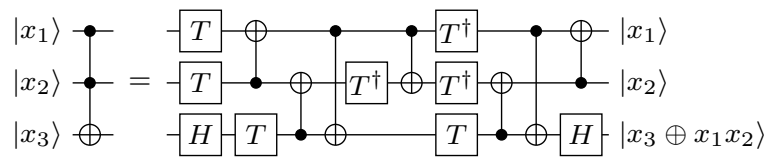

When the Toffoli gate is computed on a qubit initialized to $|0\rangle$, it can be implemented using $4 T$ gates, with a $T$-depth of 2 , and without requiring any additional qubit [17], [18]:

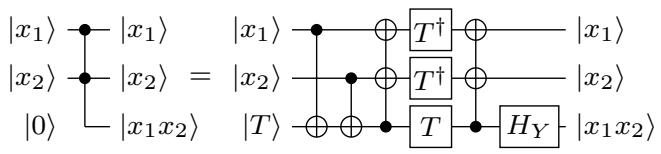

where $H_{Y}=S H$ and $|T\rangle=T H|0\rangle$. Besides, when the result of the Toffoli is uncomputed, this can be performed without the use of any $T$ gate, exploiting measurement-based uncomputation, as shown:

$$
\left.\begin{array}{r}
\left|x_{1}\right\rangle \\
\left|x_{2}\right\rangle \\
\left|x_{1} x_{2}\right\rangle
\end{array}\right\}\left\{\begin{array}{l}
\left|x_{1}\right\rangle \quad\left|x_{1}\right\rangle \\
\left|x_{2}\right\rangle=\left|x_{2}\right\rangle \\
|0\rangle \quad\left|x_{1} x_{2}\right\rangle
\end{array}\right\}
$$

Several works from the literature describe how to map larger multiple-control Toffoli gates into Clifford $+T$ gates (see, e.g., [7], [8], [19], [20]). Among them, a method proposed by Barenco et al. [21] allows us, provided an additional qubit, to map any multiple-control Toffoli gate into a sequence of 2-control Toffoli gates, which can be implemented using the optimum circuits in (1) and (2).

\section{E. Spectral operations and classification}

We define the five operations that are used to partition the set of all $n$-variable Boolean functions into equivalence classes.

Definition 4 (Spectral invariant operations [22]):

1) Swapping two variables $\left(f \stackrel{x_{i} \leftrightarrow x_{j}}{\longrightarrow} g\right.$ ).

2) Complementing a variable $\left(f \stackrel{\bar{x}_{i}}{\longrightarrow} g\right)$.

3) Complementing the function $(f \neg g)$.

4) Translational operation $\left(f \stackrel{x_{i} \oplus x_{j}}{\longrightarrow} g\right)$. It replaces one input $x_{i}$ with $x_{i} \oplus x_{j}$.

5) Disjoint translational operation $\left(f \stackrel{\oplus x_{i}}{\longrightarrow} g\right.$ ). It is obtained by XOR-ing $f$ with an input $x_{i}$.

These operations partition all $n$-variable Boolean functions into equivalence classes by means of the following equivalence relation.

Definition 5 (Spectral equivalence [23]): Two $n$-variable Boolean functions $f$ and $g$ are spectral-equivalent, if there exist operations $o_{1}, \ldots, o_{k}$ (from Definition 4) such that:

$$
f \stackrel{o_{1}}{\longrightarrow} \cdots \stackrel{o_{k}}{\longrightarrow} g .
$$

Using this equivalence relation, the set of all $n$-variable Boolean functions for $n=1,2,3,4,5,6$ collapses into just $1,2,3,8,48,150357$ equivalence classes, respectively [24], [25]. We refer the reader to the literature (e.g., [26]-[28]), for further information on spectral classification techniques.

\section{SPECTRAL EQUIVALENCE IN QUANTUM COMPILATION}

In this section, we explain how our database can be used to compile any Boolean function, by only adding Clifford gates.

Given two Boolean functions $f$ and $f^{\prime}$ such that $f \stackrel{o}{\rightarrow} f^{\prime}$ where $o$ is a spectral operation, the optimal $T$-count ( $T$-depth) for a single-target gate controlled by $f^{\prime}$ must equal the optimal $T$-count ( $T$-depth) for a single-target gate controlled by $f$. The 


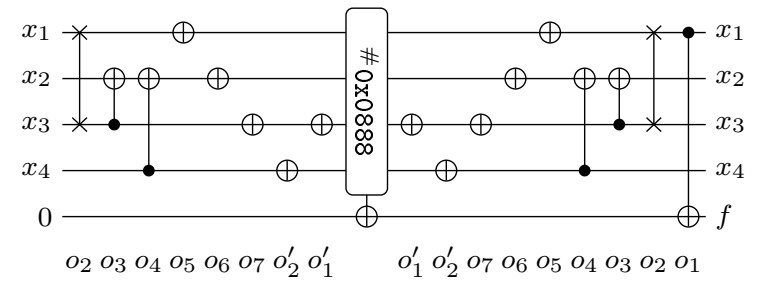

Fig. 3. Synthesized reversible circuit for the function $f$, obtained performing all the spectral operation to retrieve $f$ from the optimal implementation available in the database $f_{r}(\# 0 \times 0888)$

reason is that the five spectral operations can be implemented only using $\mathrm{X}$ and CNOT gates.

Example 2: Assume we want to map $\mathrm{T}_{f}\left(\left\{x_{1}, x_{2}, x_{3}, x_{4}\right\}, x_{5}\right)$ with $f=$ \# acab. Knowing that $f \in\left[{ }^{\#} 0888\right]$. Thus let $f_{r}=\#_{0888}$ be the database entry for this class. The spectral canonization algorithm in [22] finds the operation sequences to transform $f$ and $f_{r}$ in a canonical representative function of the equivalent class (\#8880). Respectively, $o_{1}=\oplus x_{1}, o_{2}=x_{1} \leftrightarrow x_{3}, o_{3}=x_{2} \oplus x_{3}, o_{4}=$ $x_{2} \oplus x_{4}, o_{5}=\bar{x}_{1}, o_{6}=\bar{x}_{2}, o_{7}=\bar{x}_{3}$ and $o_{1}^{\prime}=\bar{x}_{3}, o_{2}^{\prime}=\bar{x}_{4}$. We obtain a circuit as illustrated in Fig. 3. First the operations $o_{1}^{\prime}, o_{2}^{\prime}$ are applied to transform $f_{r} \rightarrow \# 8880$ then $o_{7}, \ldots, o_{1}$ to transform $\# 8880 \rightarrow f$.

The database can be used in combination with decomposition-based compilation techniques, as the look-up table (LUT) based method proposed in [10]. This method takes a logic network with an arbitrary number of inputs and decomposes it in sub-networks with maximum $k$ inputs, using $k$-LUT-based decomposition. The algorithm can be extended to fetch implementations for each sub-network from the proposed database. As a consequence, any improvement in the database would positively affect the result of the compilation.

\section{ALGORITHMS FOR THE SYNTHESIS OF SINGLE-TARGET GATES}

This section presents three different compilation methods. Each one has been developed to minimize a different cost function for fault-tolerant quantum computing: the $T$-count, the $T$-depth, and the number of qubits.

\section{A. An XAG-based algorithm to optimize T-count}

The algorithm that we employ to generate circuits with minimal $T$-count is a constructive hierarchical method based on Xor-And-inverter Graphs (XAGs) [29]. This compilation algorithm is capable of generating a quantum circuit using $O(N)$ qubits and $O(N)$ gates, where $N$ is the size of the graph. The method guarantees an upper bound on the $T$-count, which depends on the multiplicative complexity $\tilde{c}$ of the Boolean function representation. This is the number of AND nodes in the network. In particular, the $T$-count is at most equal to $4 \times \tilde{c}$. The algorithm takes advantage of the low T-count implementation of the AND function proposed by [17], [18], and shown in (2), (3).

\section{B. An XAG-based algorithm to optimize T-depth}

We propose a hierarchical constructive method that aims to minimize the circuit $T$-depth and uses XAGs as inputs. We exploit an AND gate implementation with $T$-depth $=1$, which combines the AND circuit from [18] and the $T$-depth one Toffoli gate implementation in [19]. The circuit requires one extra qubit with respect to the implementation in (2):

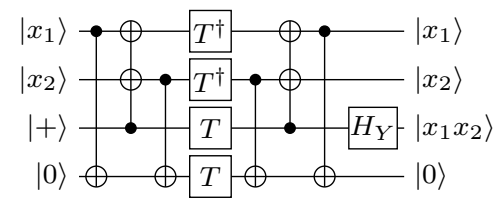

where $|+\rangle=H|0\rangle$.

Given an XAG with $d$ levels containing at least one AND node, our algorithm would generate circuits with $T$-depth equal to $d$. As a consequence, depth optimization may be applied to minimize the resulting $T$-depth. Our synthesis method is based on the fact that each AND node is driven by two multi-input parity functions, which can be computed in-place using CNOT gates. It proceeds level by level and in topological order, and:

1) finds the parity functions that are input to AND nodes;

2) computes the parity functions in-place using CNOT gates;

3 ) if the function is input to more AND nodes, uses a CNOT to copy it on a new qubit initialized to $|0\rangle$;

4) implements inversions using $X$ gates;

5) implements all AND nodes in the level using (4).

By copying some of the qubits, we enable parallel computation of all the AND nodes in one level.

\section{An ESOP-based algorithm to optimize the number of qubits}

To generate the database entries optimized for the number of qubits, we select an algorithm that generates a Toffoli network without adding any additional line. The algorithm builds a single-target gate controlled by the given Boolean function. Then, it performs the ESOP-based decomposition of the single-target gate into a cascade of Toffoli gates. Finally, each multiple-control Toffoli gate is mapped into 2-control Toffoli using Barenco decomposition [21] and in Clifford $+T$ using the methods described in Section II-D. The algorithm for ESOP synthesis that we apply is a portfolio method proposed in [30]. It selects the best ESOP-decomposition strategy between Positive Polarity Reed Muller (PPRM) [31], Pseudo-Kronecker Reed Muller (PKRM) [32], and the exact method proposed in the same publication.

\section{DATABASE OF OPTIMAL QUANTUM CIRCUITS}

We run the three compilation algorithms described in Section IV on all the spectral-equivalent class representatives of 4- and 5-input Boolean functions. Results are presented in Table I. It is clear that each algorithm succeeds in optimizing its respective cost function. To synthesize the all benchmark, the ESOP-based method uses a total of 345 qubits, against 475 and 625 qubits required by the other methods. The XAG method that targets $T$-count optimization achieves a total of $712 T$ gates, against the 10913 produced by the ESOP-based method. Besides, the XAG-based method for $T$-depth optimization achieves the same $T$ count as the previous method (712) but reduces the $T$-depth from 220 to 143, paying the price of additional Cliffords and qubits. Note that, when measurement-based uncomputation is used, the number of 
TABLE I

\begin{tabular}{|c|c|c|c|c|c|c|c|c|c|c|c|c|}
\hline \multirow[b]{2}{*}{ truth table } & \multicolumn{4}{|c|}{ optimal num. of qubits } & \multicolumn{4}{|c|}{ optimal $T$-count } & \multicolumn{4}{|c|}{ optimal $T$-depth } \\
\hline & cliffords & qubits & T-count & T-depth & cliffords & qubits & T-count & T-depth & cliffords & qubits & T-count & T-depth \\
\hline$\#_{8000}$ & 120 & 6 & 70 & 37 & 51 & 7 & 12 & 4 & 81 & 11 & 12 & 3 \\
\hline \#8080 & 48 & 5 & 28 & 19 & 23 & 6 & 8 & 3 & 29 & 8 & 8 & 2 \\
\hline$\#_{0888}$ & 132 & 6 & 77 & 42 & 47 & 7 & 12 & 4 & 85 & 10 & 12 & 3 \\
\hline \# 8888 & 12 & 5 & 7 & 5 & 9 & 5 & 4 & 2 & 17 & 7 & 4 & 1 \\
\hline$\#_{7080}$ & 60 & 5 & 35 & 24 & 25 & 6 & 8 & 3 & 45 & 9 & 8 & 2 \\
\hline$\#_{7880}$ & 182 & 6 & 105 & 55 & 70 & 8 & 12 & 4 & 89 & 11 & 12 & 3 \\
\hline$\#_{7888}$ & 24 & 5 & 14 & 9 & 22 & 7 & 8 & 2 & 35 & 8 & 8 & 1 \\
\hline \#6ac06ac0 & 24 & 6 & 14 & 9 & 26 & 8 & 8 & 2 & 31 & 9 & 8 & 1 \\
\hline${ }^{\#} 6 \mathrm{ac} 8 \mathrm{e} 000$ & 720 & 7 & 413 & 210 & 125 & 10 & 16 & 5 & 162 & 15 & 16 & 2 \\
\hline \#80008000 & 120 & 6 & 70 & 37 & 43 & 8 & 12 & 4 & 85 & 12 & 12 & 3 \\
\hline \#80808080 & 48 & 6 & 28 & 19 & 19 & 7 & 8 & 3 & 33 & 9 & 8 & 2 \\
\hline \# 88808000 & 582 & 7 & 336 & 159 & 72 & 9 & 12 & 4 & 99 & 11 & 12 & 3 \\
\hline \# 88808080 & 314 & 7 & 182 & 92 & 61 & 9 & 16 & 4 & 77 & 12 & 16 & 3 \\
\hline \# 88808880 & 170 & 6 & 98 & 50 & 65 & 8 & 12 & 4 & 97 & 11 & 12 & 3 \\
\hline \# 88888888 & 12 & 6 & 7 & 5 & 9 & 6 & 4 & 2 & 13 & 8 & 4 & 1 \\
\hline \# a8808000 & 848 & 7 & 490 & 232 & 110 & 10 & 16 & 5 & 155 & 13 & 16 & 3 \\
\hline \# a8808080 & 292 & 6 & 168 & 88 & 53 & 8 & 12 & 4 & 85 & 11 & 12 & 3 \\
\hline \# a8808880 & 436 & 7 & 252 & 123 & 78 & 10 & 16 & 5 & 119 & 12 & 16 & 3 \\
\hline \# a880a880 & 148 & 6 & 84 & 47 & 29 & 7 & 8 & 3 & 49 & 10 & 8 & 2 \\
\hline \# a8888880 & 548 & 7 & 315 & 160 & 57 & 8 & 12 & 4 & 89 & 11 & 12 & 3 \\
\hline \# a888a080 & 258 & 6 & 147 & 74 & 59 & 8 & 12 & 4 & 85 & 11 & 12 & 3 \\
\hline \# a8e0c800 & 560 & 7 & 322 & 156 & 110 & 10 & 16 & 5 & 135 & 12 & 16 & 3 \\
\hline \# aa808080 & 360 & 7 & 210 & 105 & 57 & 9 & 16 & 4 & 73 & 12 & 16 & 3 \\
\hline$\#_{\mathrm{b} 884 \mathrm{a} 880}$ & 686 & 7 & 392 & 188 & 82 & 9 & 12 & 4 & 101 & 11 & 12 & 3 \\
\hline${ }^{\#} \mathrm{bc} 88 \mathrm{a} 080$ & 709 & 7 & 399 & 192 & 104 & 10 & 16 & 5 & 137 & 13 & 16 & 3 \\
\hline${ }^{\#} \mathbf{e 0}$ a 8 c880 & 240 & 6 & 140 & 75 & 86 & 10 & 16 & 5 & 107 & 13 & 16 & 2 \\
\hline \# e1808880 & 372 & 6 & 217 & 117 & 86 & 9 & 12 & 4 & 113 & 12 & 12 & 3 \\
\hline \# e8808000 & 798 & 6 & 448 & 226 & 69 & 8 & 12 & 4 & 105 & 12 & 12 & 3 \\
\hline${ }^{\#}$ e8808002 & 721 & 7 & 420 & 222 & 92 & 10 & 16 & 5 & 113 & 12 & 16 & 3 \\
\hline \# e8808080 & 484 & 7 & 280 & 142 & 98 & 10 & 16 & 4 & 127 & 13 & 16 & 3 \\
\hline${ }^{\#}$ e8808880 & 410 & 6 & 238 & 120 & 82 & 9 & 12 & 4 & 113 & 12 & 12 & 3 \\
\hline \# e880a880 & 414 & 7 & 238 & 120 & 92 & 10 & 16 & 5 & 125 & 12 & 16 & 3 \\
\hline${ }^{\#}$ e880e880 & 250 & 6 & 140 & 79 & 98 & 9 & 12 & 4 & 125 & 12 & 12 & 3 \\
\hline \# e8818880 & 974 & 7 & 560 & 259 & 84 & 10 & 16 & 4 & 101 & 12 & 16 & 3 \\
\hline${ }^{\#}$ e881e880 & 504 & 7 & 287 & 143 & 121 & 10 & 16 & 5 & 154 & 13 & 16 & 3 \\
\hline \# e8888880 & 526 & 7 & 301 & 151 & 103 & 10 & 16 & 5 & 140 & 13 & 16 & 3 \\
\hline \# e8a08880 & 524 & 7 & 301 & 146 & 118 & 10 & 16 & 5 & 143 & 13 & 16 & 3 \\
\hline \# e8c0a880 & 268 & 6 & 147 & 79 & 86 & 10 & 16 & 5 & 123 & 12 & 16 & 3 \\
\hline${ }^{\#}$ e9a0c088 & 468 & 7 & 266 & 134 & 122 & 10 & 16 & 5 & 167 & 13 & 16 & 3 \\
\hline \# e9c0a880 & 540 & 7 & 308 & 152 & 124 & 10 & 16 & 5 & 153 & 13 & 16 & 3 \\
\hline \# ea808080 & 216 & 6 & 126 & 70 & 56 & 9 & 12 & 3 & 75 & 12 & 12 & 2 \\
\hline \# eca08880 & 414 & 6 & 238 & 120 & 113 & 10 & 16 & 5 & 150 & 13 & 16 & 3 \\
\hline${ }^{\#} \mathbf{f} 8808880$ & 528 & 7 & 308 & 155 & 80 & 10 & 16 & 4 & 117 & 13 & 16 & 3 \\
\hline${ }^{\#} \mathbf{f} 8888880$ & 594 & 7 & 343 & 168 & 78 & 9 & 12 & 4 & 105 & 12 & 12 & 3 \\
\hline${ }^{\#}$ f ca08880 & 578 & 7 & 329 & 155 & 122 & 10 & 16 & 5 & 155 & 13 & 16 & 3 \\
\hline${ }^{\#} 2888 \mathrm{a} 000$ & 96 & 6 & 56 & 33 & 45 & 8 & 12 & 3 & 65 & 11 & 12 & 2 \\
\hline \#6ac8e240 & 198 & 6 & 112 & 59 & 74 & 10 & 16 & 5 & 111 & 12 & 16 & 3 \\
\hline$\#_{78888888}$ & 60 & 6 & 35 & 19 & 56 & 9 & 12 & 4 & 75 & 13 & 12 & 2 \\
\hline \# 80000000 & 264 & 7 & 154 & 78 & 43 & 9 & 16 & 4 & 57 & 11 & 16 & 3 \\
\hline${ }^{\#} 80808000$ & 316 & 7 & 182 & 91 & 59 & 9 & 16 & 4 & 73 & 11 & 16 & 3 \\
\hline \# 88888880 & 282 & 7 & 161 & 82 & 58 & 10 & 16 & 4 & 75 & 11 & 16 & 3 \\
\hline \# e9808080 & 204 & 6 & 119 & 66 & 57 & 8 & 12 & 4 & 101 & 13 & 12 & 2 \\
\hline \# eac86240 & 148 & 6 & 84 & 47 & 44 & 9 & 12 & 4 & 67 & 11 & 12 & 3 \\
\hline \# ee 84 a060 & 198 & 6 & 112 & 61 & 90 & 10 & 16 & 5 & 119 & 15 & 16 & 2 \\
\hline Total & 19002 & 345 & 10913 & 5506 & 3842 & 475 & 712 & 220 & 5260 & 625 & 712 & 143 \\
\hline
\end{tabular}

Clifford gates varies according to the probabilistic outcomes of the measurements.

\section{CONCLUSIONS}

We present a database containing automatically generated quantum circuits for all Boolean functions up to 5 inputs. We consider the specifications of fault-tolerant quantum computing: low $T$-count, low $T$-depth, and low number of qubits. To generate the database, we combine optimal reversible gates implementations with compilation methods in the literature.
Furthermore, we propose a new XAG-based synthesis strategy for $T$-depth optimization. The database can be used to compile larger Boolean functions if combined with hierarchical methods. In this direction, we are working on extending the database to 6-input functions. The database is available at https://github.com/gmeuli/stg-benchmark and can serve as benchmark for new synthesis and optimization algorithms.

\section{Acknowledgments}

This research was supported by the Swiss National Science Foundation (200021-169084 MAJesty). 


\section{REFERENCES}

[1] C. Nay, "IBM unveils world's first integrated quantum computing system for commercial use," IBM Research Communications, 2019.

[2] F. Arute, K. Arya, R. Babbush, D. Bacon, J. C. Bardin, R. Barends, R. Biswas, S. Boixo, F. G. Brandao, D. A. Buell et al., "Quantum supremacy using a programmable superconducting processor," Nature, vol. 574, no. 7779, pp. 505-510, 2019.

[3] P. Ball, "First commercial ion-based quantum computer built," Physics World, vol. 32, no. 2, pp. 5-5, feb 2019.

[4] S. Debnath, N. M. Linke, C. Figgatt, K. A. Landsman, K. Wright, and C. Monroe, "Demonstration of a small programmable quantum computer with atomic qubits," Nature, vol. 536, pp. 63-66, 2016.

[5] P. J. J. O'Malley et al., "Scalable quantum simulation of molecular energies," Physical Review X, vol. 6, p. 031007, 2016.

[6] E. A. Martinez, C. A. Muschik, P. Schindler, D. Nigg, A. Erhard, M. Heyl, P. Hauke, M. Dalmonte, T. Monz, P. Zoller, and R. Blatt, "Real-time dynamics of lattice gauge theories with a few-qubit quantum computer," Nature, vol. 534, pp. 516-519, 2016.

[7] D. Maslov, "Advantages of using relative-phase Toffoli gates with an application to multiple control Toffoli optimization," Physical Review A, vol. 93, p. 022311, 2016.

[8] M. Amy, D. Maslov, M. Mosca, and M. Roetteler, "A meet-in-the-middle algorithm for fast synthesis of depth-optimal quantum circuits," IEEE Trans. on CAD of Integrated Circuits and Systems, vol. 32, no. 6, pp. 818-830, 2013.

[9] M. Amy, D. Maslov, and M. Mosca, "Polynomial-time T-depth optimization of Clifford $+T$ circuits via matroid partitioning," IEEE Trans. on CAD of Integrated Circuits and Systems, vol. 33, no. 10, pp. 1476-1489, 2014.

[10] G. Meuli, M. Soeken, M. Roetteler, and G. De Micheli, "ROS: Resource constrained oracle synthesis for quantum circuits," in Quantum Physics and Logic, 2019.

[11] G. Bioul, M. Davio, and J.-P. Deschamps, "Minimization of ring-sum expansions of Boolean functions," Philips Research Reports, vol. 28, pp. 17-36, 1973.

[12] A. Mishchenko and M. A. Perkowski, "Fast heuristic minimization of exclusive-sum-of-products," in Reed-Muller Workshop, 2001.

[13] S. Stergiou, K. Daskalakis, and G. K. Papakonstantinou, "A fast and efficient heuristic ESOP minimization algorithm," in ACM Great Lakes Symposium on VLSI, 2004, pp. 78-81.

[14] T. Sasao, "AND-EXOR expressions and their optimization," in Logic Synthesis and Optimization, T. Sasao, Ed. Kluwer Academic, 1993.

[15] M. A. Nielsen and I. L. Chuang, Quantum Computation and Quantum Information. Cambridge University Press, 2000.

[16] D. Gosset, V. Kliuchnikov, M. Mosca, and V. Russo, "An algorithm for the T-count," Quantum Information and Computation, vol. 14, no. 15-16, pp. 1261-1276, 2014.

[17] C. Jones, "Low-overhead constructions for the fault-tolerant Toffoli gate," Physical Review A, vol. 87, no. 2, p. 022328, 2013.

[18] C. Gidney, "Halving the cost of quantum addition," Quantum, vol. 2, no. 74, pp. 10-22331, 2018.

[19] P. Selinger, "Quantum circuits of T-depth one," Physical Review A, vol. 87, p. 042302, 2013.

[20] N. Abdessaied, M. Amy, M. Soeken, and R. Drechsler, "Technology mapping of reversible circuits to Clifford $+T$ quantum circuits," in Int'l Symp. on Multiple-Valued Logic, 2016, pp. 150-155.

[21] A. Barenco, C. H. Bennett, R. Cleve, D. P. DiVincenzo, N. Margolus, P. Shor, T. Sleator, J. A. Smolin, and H. Weinfurter, "Elementary gates for quantum computation," Physical Review A, vol. 52, no. 5, p. 3457 , 1995.

[22] C. R. Edwards, "The application of the Rademacher-Walsh transform to Boolean function classification and threshold logic synthesis," IEEE Trans. on Computers, vol. 24, no. 1, pp. 48-62, 1975.

[23] R. J. Lechner, "Harmonic analysis of switching functions," in Recent Developments in Switching Theory, A. Mukhopadhyay, Ed. Academic Press, 1971, pp. 121-228.

[24] E. R. Berlekamp and L. R. Welch, "Weight distributions of the cosets of the $(32,6)$ Reed-Muller code," IEEE Trans. on Information Theory, vol. 18, no. 1, pp. 203-207, 1972.

[25] J. A. Maiorana, "A classification of the cosets of the Reed-Muller code $\mathcal{R}(1,6), "$ Mathematics of Computation, vol. 57, no. 195, pp. 403-414, 1991.

[26] J. L. Walsh, "A closed set of normal orthogonal functions," American Journal of Mathematics, vol. 45, no. 1, pp. 5-24, 1923.
[27] M. A. Thornton, R. Drechsler, and D. M. Miller, "Computation of spectral coefficients," in Spectral Techniques in VLSI CAD. Springer, 2001, pp. $83-116$.

[28] S. L. Hurst, D. M. Miller, and J. C. Muzio, "Spectral techniques in digital logic," 1985.

[29] G. Meuli, M. Soeken, E. Campbell, M. Roetteler, and G. De Micheli, "The role of multiplicative complexity in compiling low T-count oracle circuits," arXiv preprint arXiv:1908.01609, 2019.

[30] G. Meuli, B. Schmitt, R. Ehlers, H. Riener, and G. De Micheli, "Evaluating ESOP optimization methods in quantum compilation flows," in Reversible Computation, 2019.

[31] I. Zhegalkin, "The technique of calculation of statements in symbolic logic," Mathe, Sbornik, vol. 34, pp. 9-28, 1927.

[32] R. Drechsler, "Pseudo-Kronecker expressions for symmetric functions," IEEE Transactions on Computers, vol. 48, no. 9, pp. 987-990, 1999. 